\title{
Strategies for Molecular Imaging of Epidermal Growth Factor Receptor Tyrosine Kinase in Cancer
}

\author{
Eyal Mishani and Aviv Hagooly \\ Cyclotron Unit, Department of Nuclear Medicine, Hadassah Hebrew University Hospital, Jerusalem, Israel
}

\begin{abstract}
A wealth of research has focused on developing targeted cancer therapies by specifically inhibiting epidermal growth factor receptor tyrosine kinase (EGFR-TK). However, the outcome of most EGFR-TK-targeted drugs that were approved by the Food and Drug Administration or entered clinical trials has been only moderate. Enhancement of EGFR-targeted therapy hinges on a reliable in vivo quantitative molecular imaging method. Such a method would enable monitoring of receptor drug binding and receptor occupancy in vivo; determination of the duration of EGFR inhibition in vivo; and, potentially, identification of a primary or secondary mutation in EGFR leading to drug interaction or loss of EGFR recognition by the drug. This review analyzes the most recent strategies to visualize and quantify EGFR-TK in cancer by nuclear medicine imaging and describes future directions.
\end{abstract}

Key Words: EGFR; PET; cancer; imaging; tyrosine kinase; cetuximab; gefitinib

J Nucl Med 2009; 50:1199-1202

DOI: 10.2967/jnumed.109.062117

Because of the enhanced cell proliferation characteristic of many cancers, most of the first anticancer drugs developed were based on nonspecific agents that inhibited pathways related to RNA and DNA synthesis. This approach, although making a significant contribution at the onset, had limited ultimate success because of toxicities, side effects, and insufficient and unpredictable responses. The strategic turning point for the treatment of malignancies commenced in the mid-1990s with the discovery that mutation, constitutive activation, and aberrant expression of a set of genes (oncogenes) coding for proteins regulating cell proliferation, cell differentiation, and apoptosis were implicated in cancer pathogenesis and may serve as markers for specific and targeted treatment of cancer (1). In parallel, the emerging chemical and biochemical technologies served as essential adjuncts for anticancer drug development directed at these markers and resulted in more specific targeted approaches for treating cancer.

\footnotetext{
Received Mar. 15, 2009; revision accepted May 21, 2009.

For correspondence or reprints contact: Eyal Mishani, Department of Nuclear Medicine, Hadassah University Hospital, P.O. Box 12000, Jerusalem, Israel 91120.

E-mail: eyalmi@ekmd.huji.ac.il

Guest Editor: Caius Radu, UCLA Crump Institute

COPYRIGHT @ 2009 by the Society of Nuclear Medicine, Inc.
}

One such valid approach focused on inhibiting protein tyrosine kinases (PTKs), including the epidermal growth factor receptor tyrosine kinase (EGFR-TK), that interfere with crucial signaling pathways that are dysregulated in malignant cells $(1-4)$. The role of PTKs appears to be crucial in signal transduction pathways that regulate both intracellular signaling and multicellular communication, and regulated signaling is pivotal to the development and survival of normal and cancer cells. PTKs catalyze the transfer of phosphate in adenosine triphosphate (ATP) to specific tyrosine residues within proteins, thereby altering their structure and function. PTKs are involved in multiple processes such as metabolism, cell movement, cell proliferation, angiogenesis, and inhibition of apoptosis. PTK dysregulation, such as overexpression of the TK receptor and its ligand, dimerization of PTK receptors (RTKs) or cellular PTKs with a related protein, or various mutations in the PTK itself, leads to enhanced and constant stimulation, culminating in various diseases, including cancer (1-4). Hence, PTKs, whether receptors or cellular proteins, have become valid targets to combat cancer, and the epidermal growth factor family of membrane receptors (a member of subclass I of the RTK superfamily) is one of the most relevant markers in this class. EGFR comprises 3 regions: an extracellular binding domain, a single hydrophobic transmembrane region, and the intracellular domain that harbors TK activity. EGFR activation normally occurs by a 3-step mechanism. The first step is binding of specific ligands such as EGF, amphiregulin, or TGF- $\alpha$ to the extracellular ligand binding domain of the receptor. The second step is dimerization of the ligand-bound receptor with one of the subclass I RTKs (HER1-HER4), leading to the third step, intracellular binding of ATP molecules and autophosphorylation of specific tyrosine residues within the TK domain of the receptor. These phosphotyrosine moieties serve as docking sites for further downstream signal transduction molecules, thus resulting in the various processes mentioned above.

\section{EGFR-TARGETED THERAPY}

Two approaches were attempted to inhibit EGFR in cancer. The first was based on small organic molecules targeting the internal TK domain and inhibiting autophosphorylation, The second approach used antibodies targeting the extracellular ligand binding site and inhibiting cancer cell growth by any of several direct processes, including interruption of EGFR signaling by blockage of its ligand binding, leading to inhibition of cell cycle progression or DNA repair, deceleration of angiogenesis or induction of apoptosis, or indirect processes mediated by the immune system. However, most of these drugs that have been approved by the Food and Drug 
Administration or have entered clinical trials have not yielded the predicted positive outcome (5-7). For example, the small organic molecules directed at the tyrosine kinase domain of the receptor, gefitinib and erlotinib, which exhibited promising results in preclinical studies, had only a moderate outcome clinically and were effective in only a subset of non-small cell lung cancer patients who expressed well-defined EGFR-activating mutations (8-10). Moreover, patients who initially responded to these drugs occasionally became resistent to therapy $(11,12)$. Another example is cetuximab, an IgG1 class $\mathrm{mAb}$ that had positive clinical results in head and neck cancer overexpressing EGFR but insufficient effects in EGFRoverexpressing breast cancer and only a moderate response in colon cancer regardless of EGFR expression. Thus, patient response to cetuximab could not be predicted solely on the basis of EGFR expression, although it is a prerequisite for initiation of treatment (13-15). These results could be due to several factors, including inappropriate selection of potential responders in terms of EGFR expression, insufficient inhibition of the receptor because of an inappropriate dosage schedule, resistance to a specific drug because of a secondary mutation acquired during treatment, loss of the survival function role of EGFR, and development of other pathways as an escape mechanism for cancer cell survival. Thus, better predictors are needed for proper selection of responders, prediction of therapeutic outcome, and guidance or monitoring of EGFR-targeted treatment.

To enhance EGFR-targeted therapies, new directions were suggested and undertaken. One new direction was therapy with multiple drugs working in synergy: one drug inhibiting EGFR activation and other drugs targeting other cancer-specific pathways, such as drugs that inhibit angiogenesis by targeting vascular endothelial growth factor and its receptor (VEGFR). Another new direction was the development of drugs targeting EGFR along with other PTKs having a role in cancer cell proliferation, such as lapatinib (Tykerb), which also targets erbB2; canertinib (CI-1033), a pan-erbB inhibitor; and semaxanib (SU5416), which also targets the VEGFR and KIT (1). A third new direction was the development of a reliable and accurate in vivo quantitative method to determine EGFR levels of expression and their role in specific tumors and metastases so as to guide and monitor customized EGFR-targeted cancer therapy. One possible approach is labeled EGFR molecular imaging agents. These will enable monitoring of receptor drug binding and receptor occupancy in vivo; determination of the duration of EGFR inhibition in vivo; and, potentially, identification of a primary or secondary mutation in the EGFR leading to drug interaction or loss of EGFR recognition by the drug.

\section{APPROACHES FOR IMAGING EGFR IN CANCER}

Before labeled biomarkers targeting saturable systems such as EGFR are developed, several crucial factors should be considered (16). Some of these are drug development prerequisites, such as the need for a tracer with high selectivity, high affinity, and stability. However, for molecular imaging agents, the need to generate quantitative high-resolution and specific images of the target brings other requirements. Although EGFR expression should be sufficiently higher in the tumor than in the surrounding area to generate clear signals, too high a concentration of the receptor (maximum number of binding sites, or $\mathrm{B}_{\max }$ ) might lead to measurements of tracer flow or permeability rather than of specific binding when a high-affinity (low dissociation constant, or $\mathrm{K}_{\mathrm{D}}$ ) tracer is used. This leads to measurement of binding potential, which is the ratio of the target concentration $\left(\mathrm{B}_{\max }\right)$ to the $\mathrm{K}_{\mathrm{D}}$ of the tracer, commonly in the range of 10-50. The specific activity of the tracer should be high enough to avoid saturation of binding sites; otherwise, under saturating conditions, it would be impossible to measure changes in the concentration of the receptor binding site. In order to achieve quantitative high-resolution images, appropriate blood clearance, biodistribution and pharmacokinetics commensurate with radionuclide half-life should yield sustained high specific binding in the targeted tumor and clearance from metabolic organs $(17,18)$.

Like the approaches used to develop EGFR-targeted drugs, approaches toward developing EGFR-labeled bioprobes were directed either at the external ligand binding domain using antibodies and Affibodies or at the internal ATP binding domain using small organic reversible and irreversible inhibitors based mainly on the anilinoquinazoline moiety (Fig. 1) (7,17-20). In the domain of reversible anilinoquinazoline chemical moieties, many drugs and their derivatives such as PD 153035, gefitinib, erlotinib, ZD 6474, and ML01 were labeled with diverse radionuclides such as ${ }^{11} \mathrm{C},{ }^{123} \mathrm{I},{ }^{125} \mathrm{I},{ }^{18} \mathrm{~F}(17,18,20-22)$, and the radiometal ${ }^{99 \mathrm{~m}} \mathrm{Tc}$ using 2 different chelators (23). Among all these labeled reversible inhibitors, ${ }^{11} \mathrm{C}$-PD153035 was the only one whose biodistribution and dosimetry were recently evaluated in healthy volunteers (22) and indicated a favorable radiation dose profile. These reversible compounds were labeled either at the functional group at the 6- or 7-position of the quinazoline ring or at the anilino moiety (Fig. 1). Most labeled inhibitors in this class had an impressive in vitro profile, including high affinity at the subnanomolar range, selectivity toward EGFR rather than toward other PTKs, stability, and specific binding to EGFR-positive tumor cells such as A431 and U87. However, in the preclinical phase, when various animal models bearing tumors such as SH-SY5Y human neuroblastoma, U87positive EGFR human glioma, and A431 were used to evaluate the potential of these labeled biomarkers in biodistribution or smallanimal PET studies, most were found to be inadequate for molecular imaging (17). They exhibited low uptake in targeted tumors (low tumor-to-blood and tumor-to-muscle activity uptake ratios) and high uptake in nontargeted tissues such as the gastrointestinal tract,

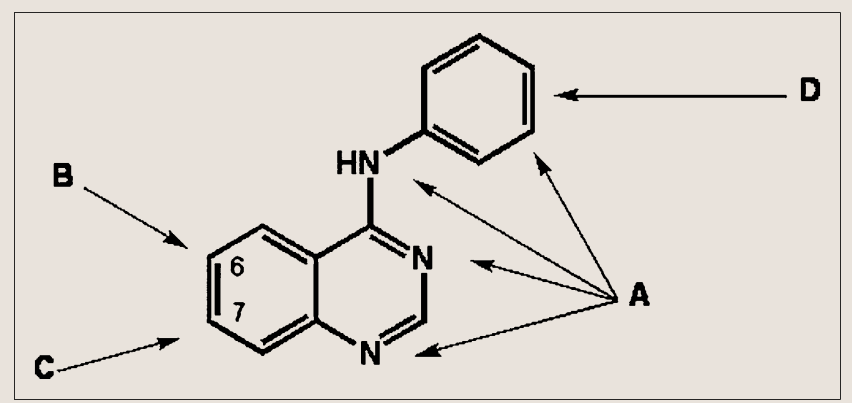

FIGURE 1. Anilinoquinazoline core structure: strategic and labeling positions. (A) The 3 nitrogen atoms and electronwithdrawing groups at meta positions of aniline are important for achieving high affinity and selectivity toward EGFR. (B) To achieve covalent bonding to cysteine-773 and form irreversible inhibitor, various chemical moieties were attached at 6-position of quinazoline ring. Some of these moieties were labeled with ${ }^{11} \mathrm{C}$. (C) Since functional group at 7-position of quinazoline ring is positioned toward exit of binding domain, it was used as anchor to attach bulky solubilizing groups such as fluorinated and free-hydroxy short-PEG chains and morpholino derivatives. Some of these groups were labeled with ${ }^{18} \mathrm{~F}$. (D) Several positions on alinino ring were used for labeling with ${ }^{18} \mathrm{~F}$ and ${ }^{124} \mathrm{I}$. 
liver, and kidney. Moreover, specific binding in tumors was not evident in blocking studies using injections of an excess of nonlabeled EGFR inhibitors before or with the labeled bioprobe. The failure of these labeled reversible inhibitors to specifically image EGFR in the preclinical phase could be attributed to their elevated $\log \mathrm{P}$ (lipophilicity), fast blood clearance, rapid metabolism; to binding competition between manifold higher levels of intracellular ATP and the labeled bioprobe; and to the inability of these types of compounds to bind properly to the nonactivated (nonphosphorylated) form of the receptor. Indeed, small-animal PET and biodistribution studies recently have shown that, compared with $\mathrm{A} 549$ and $\mathrm{NC} 1358,{ }^{11} \mathrm{C}$-erlotinib has high and sustained uptake in HCC827 tumor-bearing mice (Fig. 2). In contrast to A549 and NC1358 cells, HCC827 cells have high expression of EGFR but, more important, harbor an in-frame deletion mutation (delE746A750) in exon 19 (phosphorylated form) (21).

To enhance binding efficacy, much effort has recently been invested in the development of irreversible EGFR-TK inhibitors as drugs and imaging agents $(1,17,18)$. In addition, according to recent publications, it appears that irreversible inhibitors can circumvent the resistance of a secondary mutation to reversible drugs, thus potentially having wider applications $(1,18)$. Similarly to reversible inhibitors, these irreversible compounds are based on the anilinoquinazoline core structure but include a functional group at the 6position of the anilinoquinazoline moiety that can form covalent bonding with cysteine 773 at the ATP binding pocket. Numerous labeled irreversible EGFR inhibitors were developed with various functional groups at the 6-position, aimed at irreversible binding to the receptor, and with various functional groups at the 7-position, such as polyethylene glycol (PEG), aimed at increasing solubility, decreasing lipophilicity, and thus reducing fast blood clearance and nonspecific binding in tumors (Fig. 1) (18). These candidates were labeled with various short- and long-lived radionuclides, either at the 6- or 7-position of the quinazoline ring (Fig. 1) or at various positions at the anilino moiety, and were studied in vitro and in vivo. These biologic evaluations revealed and highlighted several crucial points with regard to the development of EGFR-TK-labeled imaging agents. In general, labeled irreversible bioprobes exhibited higher-activity uptake in tumors and much higher tumor-to-blood and tumor-to-muscle uptake ratios even in nonactivated (nonphosphorylated) tumors than did their reversible analogs. The
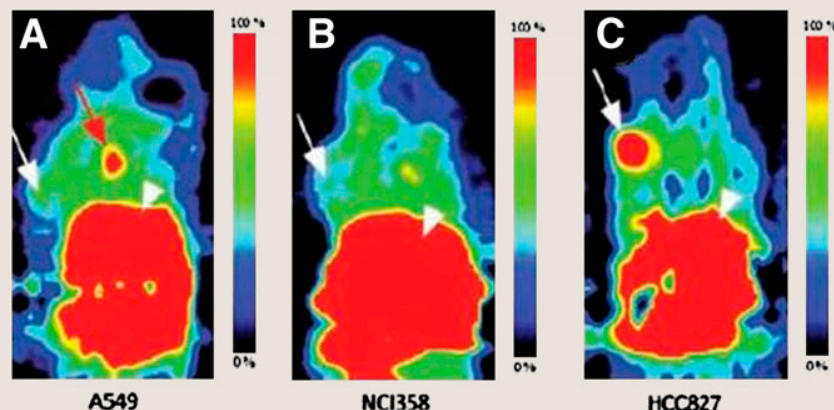

FIGURE 2. ${ }^{11} \mathrm{C}$-erlotinib small-animal PET of human lung cancer xenografts: coronal images of athymic nude mice with A549 (A), NCl358 (B), and HCC827 (C) lung cancer xenografts at left shoulder. HCC827 has high expression of EGFR and harbors in-frame deletion mutation (delE746-A750) in exon 19. Tumor location is indicated by white arrows, and liver uptake by white arrowheads. (Reprinted with permission of (21).) chemical reactive group at the 6-position aiming to form covalent binding with cysteine 773 at the ATP binding domain should be carefully designed to avoid overreactivity, fast clearance from blood, and high accumulation in metabolic organs. Currently, the dimethyl crotonylamide has been found to be the most suitable reactive group. Significant positive effects on solubility and $\log \mathrm{P}$ were obtained with the free-hydroxy PEG group as a solubilizing group at the 7-position (24). Based on biodistribution and smallanimal PET studies of these irreversible labeled bioprobes with various tumor-bearing mice, it appears that to obtain clear and elevated signals in the tumor tissue while reducing background activity levels in nontargeted tissues, the use of longer-lived isotopes is more appropriate. Two labeled EGFR bioprobes stand out over the others. The first is ${ }^{18} \mathrm{~F}-\mathrm{MLO4}$, and the second is morpholino- ${ }^{124} \mathrm{I}-$ IPQA ( $(E)$-But-2-enedioic acid [(4-(3-iodoanilino)-quinazoline-6yl]-amide-(3-morphlin-4-yl-propyl)-amide) $(17,18,20)$. Both could clearly visualize EGFR-positive tumors, and ${ }^{18} \mathrm{~F}-\mathrm{ML} 04$ had sufficient tumor-to-blood and tumor-to-muscle activity uptake ratios. The morpholino- ${ }^{124}$ I-IPQA has unique potential as a PET agent since it has shown selective and irreversible binding to the ATP binding site of the activated (phosphorylated) but not of the inactivated EGFR kinase, thus having the ability to discriminate in vivo between them (20). However, the major drawbacks of these imaging agents are their high accumulation in nontargeted tissues and, for ${ }^{18} \mathrm{~F}-\mathrm{ML} 04$, high nonspecific binding in EGFR-positive tumors. Based on the structure of ML04, a newer generation of tracers labeled with ${ }^{124}$ I was developed with free-hydroxy PEG at the 7-position and various crotonylamide groups at the 6-position (Fig. 1) $(18,24)$. In vitro studies found these agents to be potent, selective, and stable and to exhibit much higher solubility, lower lipophilicity, and slower blood clearance than do previously described labeled bioprobes. Currently, their pharmacokinetics and specific binding in different EGFR-positive tumor-bearing mice are being evaluated in biodistribution and small-animal PET studies.

For targeting the external binding domain of the receptor, labeled anti-EGFR mAbs were developed. Most were based on cetuximab conjugates (Fig. 3) (18). Conjugates such as DTPA-cetuximab, DTPA-PEG-cetuximab, 1,4,7,10-tetraazacyclododecane- $N, N^{\prime}, N^{\prime \prime}$, $N^{\prime \prime \prime}$-tetraacetic acid (DOTA)-cetuximab, cetuximab- $N$-sucDf, and cetuximab- $p$-SCN-benzyl-diethylenetriaminepentaacetic acid (DTPA) were labeled with various radionuclides, including ${ }^{64} \mathrm{Cu}$ (Fig. 3), ${ }^{88} \mathrm{Y},{ }^{111} \mathrm{In}$, and ${ }^{125} \mathrm{I}$. In general, although most of these labeled bioprobes localized specifically in tumors that overexpress EGFR, some exhibited considerable radioactivity in the liver. This limitation could be overcome either by imaging later after injection or by adding PEG chains to the conjugating chelators, as in the case of DTPA-PEG-cetuximab, which reduced high uptake in the liver (18). Lower-molecular-weight Affibodies that bind to EGFR have been developed as alternatives to radiolabeled intact mAbs as imaging agents. These Affibodies were labeled with ${ }^{125} \mathrm{I}$ using $p$-iodobenzoate or with ${ }^{111}$ In using benzyl-DTPA $(18,25)$. Currently, although some of the labeled EGFR-targeted Affibodies developed thus far have exhibited impressive tumor-to-blood uptake ratios, significantly high kidney uptake and low-to-moderate tumor uptake were evident in A431 xenograft mice.

\section{CONCLUSION}

To improve the therapeutic potential of EGFR-targeted drugs, efforts have been invested in developing labeled EGFR molecular imaging agents. The use of these selective labeled agents in nuclear medicine applications may facilitate in vivo EGFR-targeted drug 


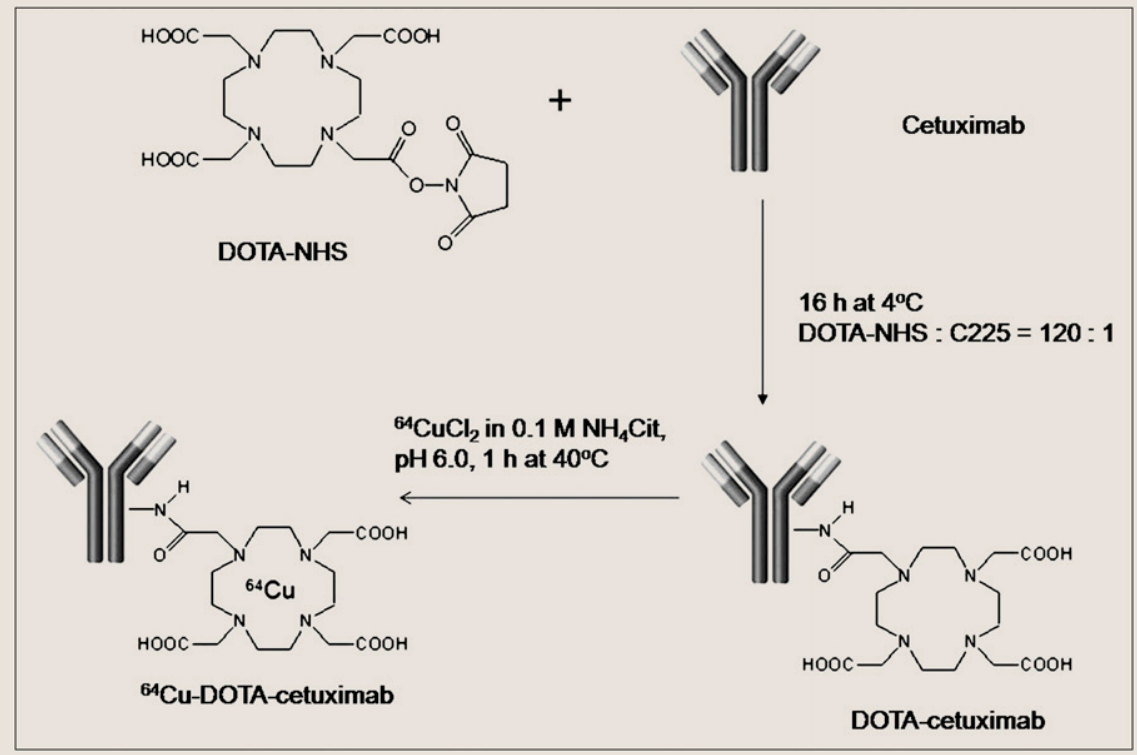

FIGURE 3. Preparation of ${ }^{64} \mathrm{Cu}-\mathrm{DOTA}$ cetuximab for PET of EGFR-positive tumors.

efficacy by noninvasively assessing the expression level of EGFR in specific tumors, guiding dosage and regime by measuring targetdrug interaction and receptor occupancy, and potentially detecting a primary or secondary mutation leading to positive response or resistance to a specific drug. In the field of labeled low-molecularweight TK reversible and irreversible inhibitors, although encouraging results were recently obtained with ${ }^{11} \mathrm{C}$-erlotinib (reversible inhibitor) when tested in a $\mathrm{HCC} 827$ xenograft that harbors an inframe deletion mutation in exon 19 , irreversible labeled compounds seem to be more promising, especially those exhibiting high solubility and lowered $\log \mathrm{P}$, and thus should also be tested in xenografts harboring the erlotinib-sensitive mutation. To obtain a high signal-to-noise ratio, longer-lived isotopes should be used so that imaging can be performed later after injection to afford washout of the high uptake from nontargeted tissues. Future trends in the field of labeled small molecules will probably include labeled inhibitors directed toward the intracellular substrate binding site rather than the ATP binding domain to avoid ATP binding competition and to obtain higher accumulation and selectivity. Although promising results were obtained with some cetuximab conjugates, especially with DOTA-cetuximab labeled with ${ }^{64} \mathrm{Cu}$, which exhibits high specificity and high accumulation in tumor, their slow clearance from the bloodstream and metabolic tissues resulted in limited imaging contrast early after injection. This limitation may be overcome with the development of labeled Affibodies or small peptides targeting the extracellular binding domain of the receptor.

\section{REFERENCES}

1. Levitzki A, Mishani E. Tyrphostins and other tyrosine kinase inhibitors. Annu Rev Biochem. 2006;75:93-109.

2. Baselga J. Targeting tyrosine kinases in cancer: the second wave. Science. 2006;312:1175-1178.

3. Ritter CA, Arteaga CL. The epidermal growth factor receptor-tyrosine kinase: a promising therapeutic target in solid tumors. Semin Oncol. 2003;30:3-11.

4. Hopfner M, Schuppan D, Scherubl H. Growth factor receptors and related signaling pathways as targets for novel treatment strategies of hepatocellular cancer. World J Gastroenterol. 2008;14:1-14.

5. Lelli G, Cataldo S, Carandina I, et al. The role of cetuximab in pre-treated refractory patients with metastatic colorectal cancer: outcome study in clinical practice. J Chemother. 2008;20:374-379.

6. Pantaleo MA, Nannini M, Lopci E, et al. Molecular imaging and targeted therapies in oncology: new concepts in treatment response assessmenta collection of cases. Int J Oncol. 2008;33:443-452.
7. Pantaleo MA, Nannini M, Maleddu A, et al. Experimental results and related clinical implications of PET detection of epidermal growth factor receptor (EGFr) in cancer. Ann Oncol. 2009;20:213-226.

8. Paez JG, Janne PA, Lee JC, et al. EGFR mutations in lung cancer: correlation with clinical response to gefitinib therapy. Science. 2004;304:1497-1500.

9. Lynch TJ, Bell DW, Sordella R, et al. Activating mutations in the epidermal growth factor receptor underlying responsiveness of non-small-cell lung cancer to gefitinib. N Engl J Med. 2004;350:2129-2139.

10. Riely GJ, Politi KA, Miller VA, Pao W. Update on epidermal growth factor receptor mutations in non-small cell lung cancer. Clin Cancer Res. 2006;12:7232-7241.

11. Pao W, Miller VA, Politi KA, et al. Acquired resistance of lung adenocarcinomas to gefitinib or erlotinib is associated with a second mutation in the EGFR kinase domain. PLoS Med. 2005;2:e73.

12. Kobayashi S, Boggon TJ, Dayaram T, et al. EGFR mutation and resistance of non-small-cell lung cancer to gefitinib. N Engl J Med. 2005;352:786-792.

13. Cunningham D, Humblet Y, Siena S, et al. Cetuximab monotherapy and cetuximab plus irinotecan in irinotecan-refractory metastatic colorectal cancer. N Engl J Med. 2004;351:337-345.

14. Bernier J. Cetuximab in the treatment of head and neck cancer. Expert Rev Anticancer Ther. 2006;6:1539-1552.

15. Chung KY, Shia J, Kemeny NE, et al. Cetuximab shows activity in colorectal cancer patients with tumors that do not express the epidermal growth factor receptor by immunohistochemistry. J Clin Oncol. 2005;23:1803-1810.

16. Mankoff DA, Link JM, Linden HM, Sundararajan L, Krohn KA. Tumor receptor imaging. J Nucl Med. 2008;49(suppl):149S-163S.

17. Mishani E, Abourbeh G. Cancer molecular imaging: radionuclide-based biomarkers of the epidermal growth factor receptor (EGFR). Curr Top Med Chem. 2007;7:1755-1772.

18. Mishani E, Abourbeh G, Eiblmaieer M, Anderson CJ. Imaging of EGFR and EGFR tyrosine kinase overexpression in tumors by nuclear medicine modalities. Curr Pharm Des. 2008;14:2983-2998.

19. Cai W, Niu G, Chen X. Multimodality imaging of the HER-kinase axis in cancer. Eur J Nucl Med Mol Imaging. 2008;35:186-208.

20. Gelovani JG. Molecular imaging of epidermal growth factor receptor expressionactivity at the kinase level in tumors with positron emission tomography. Cancer Metastasis Rev. 2008;27:645-653.

21. Memon AA, Jakobsen S, Dagnaes-Hansen F, Sorensen BS, Keiding S, Nexo E. Positron emission tomography (PET) imaging with $\left[{ }^{11} \mathrm{C}\right]$-labeled erlotinib: a microPET study on mice with lung tumor xenografts. Cancer Res. 2009;69:873-878.

22. Liu N, Li M, Li X, et al. PET-based biodistribution and radiation dosimetry of epidermal growth factor receptor-selective tracer $\left[{ }^{11} \mathrm{C}\right]-\mathrm{PD} 153035$ in humans. J Nucl Med. 2009;50:303-308.

23. Fernandes C, Santos IC, Santos I, et al. Rhenium and technetium complexes bearing quinazoline derivatives: progress towards a Tc-99m biomarker for EGFR-TK imaging. Dalton Trans. 2008;24:3215-3225.

24. Dissoki S, Eshet R, Billauer H, Mishani E. Modified PEG-anilinoquinazoline derivatives as potential EGFR PET agents. J Labelled Comp Radiopharm. 2009;52:41-52.

25. Tolmachev V, Friedman M, Sandström M, et al. Affibody molecules for epidermal growth factor receptor targeting in vivo: aspects of dimerization and labeling chemistry. J Nucl Med. 2009;50:274-283. 